\title{
Improved broadband and quasi- omnidirectional anti-reflection properties with biomimetic silicon nanostructures
}

\author{
YI-FAN HUANG ${ }^{1,2}$, SUROJIT CHATTOPADHYAY ${ }^{3 *}$, YI-JUN JEN ${ }^{1}$, CHENG-YU PENG ${ }^{1}$, TZE-AN LIU4 \\ YU-KUEI HSU², CI-LING PAN ${ }^{5}$, HUNG-CHUN L06, CHIH-HSUN HSU7, YUAN-HUEI CHANG', \\ CHIH-SHAN LEE ${ }^{8}$, KUEI-HSIEN CHEN² AND LI-CHYONG CHEN*
}

\author{
'Department of Electro-Optical Engineering, National Taipei University of Technology, Taipei 106, Taiwan \\ ${ }^{2}$ Institute of Atomic and Molecular Sciences, Academia Sinica, Taipei 106, Taiwan \\ ${ }^{3}$ Department of Electrical Engineering, National Chung Hsing University, Taichung 402, Taiwan \\ ${ }^{4}$ Center for Measurement Standards, Industrial Technology Research Institute, Hsinchu 300, Taiwan \\ ${ }^{5}$ Department of Photonics and Institute of Electro-Optical Engineering, National Chiao Tung University, Hsinchu 300, Taiwan \\ ${ }^{6}$ Department of Materials Science and Engineering, National Chiao Tung University, Hsinchu 300, Taiwan \\ ${ }^{7}$ Center for Condensed Matter Sciences, National Taiwan University, Taipei 106, Taiwan \\ ${ }^{8}$ Department of Physics National Taiwan University, Taipei 106, Taiwan \\ *e-mail: sur@dragon.nchu.edu.tw; chenlc@ccms.ntu.edu.tw
}

Nature routinely produces nanostructured surfaces with useful properties $^{1-4}$, such as the self-cleaning lotus leaf 5 , the colour of the butterfly wing ${ }^{6}$, the photoreceptor in brittlestar ${ }^{7}$ and the anti-reflection observed in the moth $\mathrm{eye}^{8}$. Scientists and engineers have been able to mimic some of these natural structures in the laboratory and in real-world applications ${ }^{9-12}$. Here, we report a simple aperiodic array of silicon nanotips on a 6-inch wafer with a sub-wavelength structure that can suppress the reflection of light at a range of wavelengths from the ultraviolet, through the visible part of the spectrum, to the terahertz region. Reflection is suppressed for a wide range of angles of incidence and for both s- and p-polarized light. The antireflection properties of the silicon result from changes in the refractive index caused by variations in the height of the silicon nanotips, and can be simulated with models that have been used to explain the low reflection from moth eyes ${ }^{8,13,14}$. The improved anti-reflection properties of the surfaces could have applications in renewable energy and electro-optical devices for the military.

In biomimetics it is critical to define both the morphology and the dimensions of the nanostructure to produce the desired functionality ${ }^{1,3,4}$. For instance, it can be shown that the optimized anti-reflection properties of the moth eye are not only due to the two-dimensional sub-wavelength structures, but are also a result of the continuously tapered morphology observed on the surface of the eye. In addition to generating a superior graded refractive index profile between the air and the surface (of the eye or substrate), this combination also exhibits an optimum anti-reflection property over a wide range of angles and wavelengths ${ }^{14-16}$. Reflection losses have been suppressed at short wavelengths (below $2.5 \mu \mathrm{m})^{17}$, but there have been few reports of anti-reflection structures that work at long wavelengths (above $10 \mu \mathrm{m})^{18}$.
There are a variety of techniques for fabricating anti-reflection nanostructures ${ }^{17,19}$, but some involve conventional wet etching ${ }^{20}$, producing porous surfaces that can be rather fragile, and other approaches involve lithography, which is often either too expensive or unsuitable for making sub-wavelength structures with high aspect ratios over large areas ${ }^{21-23}$. These difficulties have hindered the use of anti-reflection nanostructures in applications such as solar cells, electro-optical devices and sensors $^{24-26}$. This article tackles the challenge of taking the shine off silicon with the help of a large-area biomimetic silicon nanotip (SiNTs) structure. We show that the aperiodic or randomly grown SiNTs can achieve levels of reflection that are lower than those demonstrated by conventional multi-material films over a broad range of wavelengths ${ }^{17,18,21}$ and angles of incidence (AOI). Moreover, performance is almost independent of the polarization of the incident light.

Figure 1a compares an optical photograph of a 28 -square-inch (6-inch diameter) single-crystalline silicon wafer (with a polished grey look) and a wafer composed of SiNTs ${ }^{27,28}$ (with a dark black look), indicating the strong anti-reflection property for the latter. To date, to the best of our knowledge, the largest area of subwavelength structures displaying anti-reflection properties is about 2.5 square inches ${ }^{23}$. Geometric features of the SiNTs (ref. 29) were characterized by an apex diameter in the range $\sim 3-5 \mathrm{~nm}$, a base diameter of $\sim 200 \mathrm{~nm}$, lengths from $\sim 1,000$ to $16,000 \mathrm{~nm}$, and a tip density of $\sim 6 \times 10^{9} \mathrm{~cm}^{-2}$ (Fig. 1b,c). The possible application of SiNTs in photovoltaics, for which absorption is a more relevant property, is demonstrated through a marked gain in absorption, mostly at the expense of reflection, over the UV-VIS-NIR region (Fig. 1d).

In the past, it has been difficult to lower the reflectance in microstructured silicon below $1 \%$ in the UV range, and it has shown an average reflectance of $\sim 1-5 \%$ in the visible region ${ }^{19}$. 

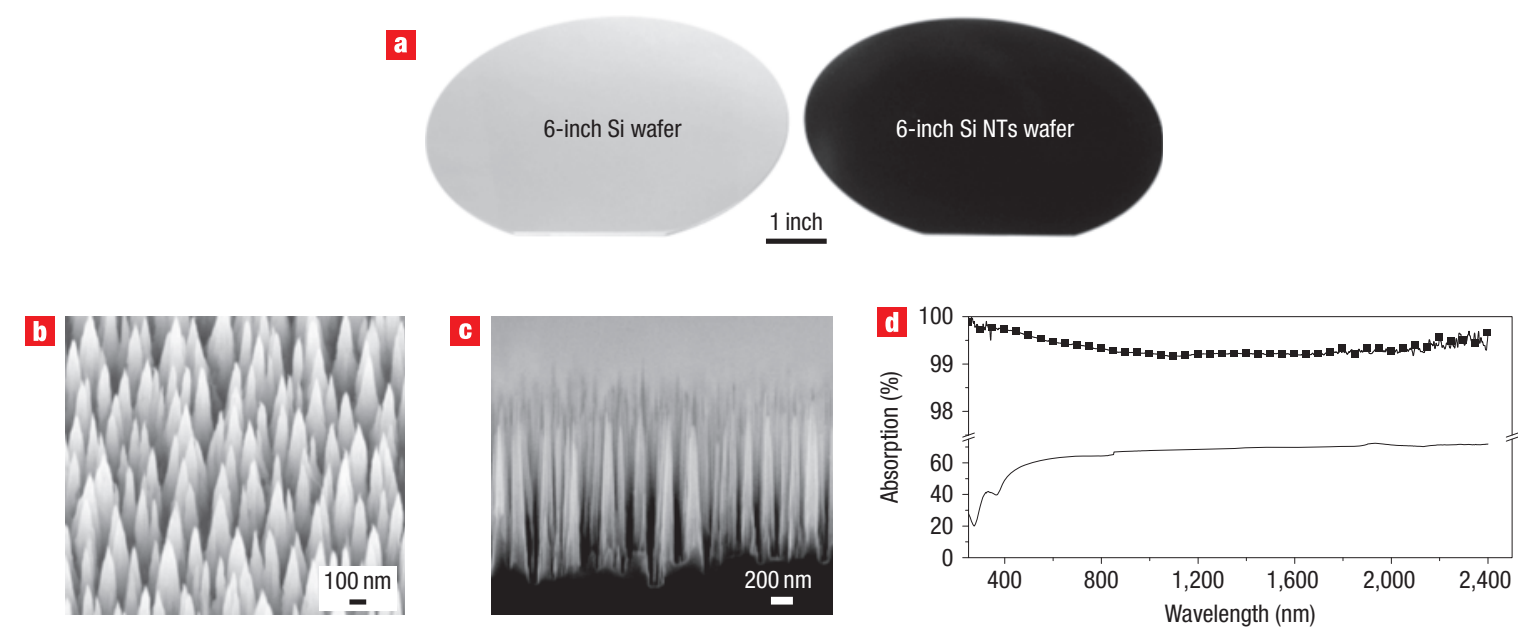

Figure 1 Photographic and scanning electron microscopy (SEM) images of a 6-inch silicon nanotips (SiNTs) wafer. a, Photographic images showing the 6-inch polished silicon wafer (left) and the wafer coated with SiNTs (right). b,c, SEM images showing a tilted top view (b) and a cross-sectional view (c) of SiNTs of length $1,600 \mathrm{~nm}$. d. Comparative absorption spectrum of the SiNTs shown in c (filled squares) and crystalline silicon (solid line) over the UV-VIS-NIR region.
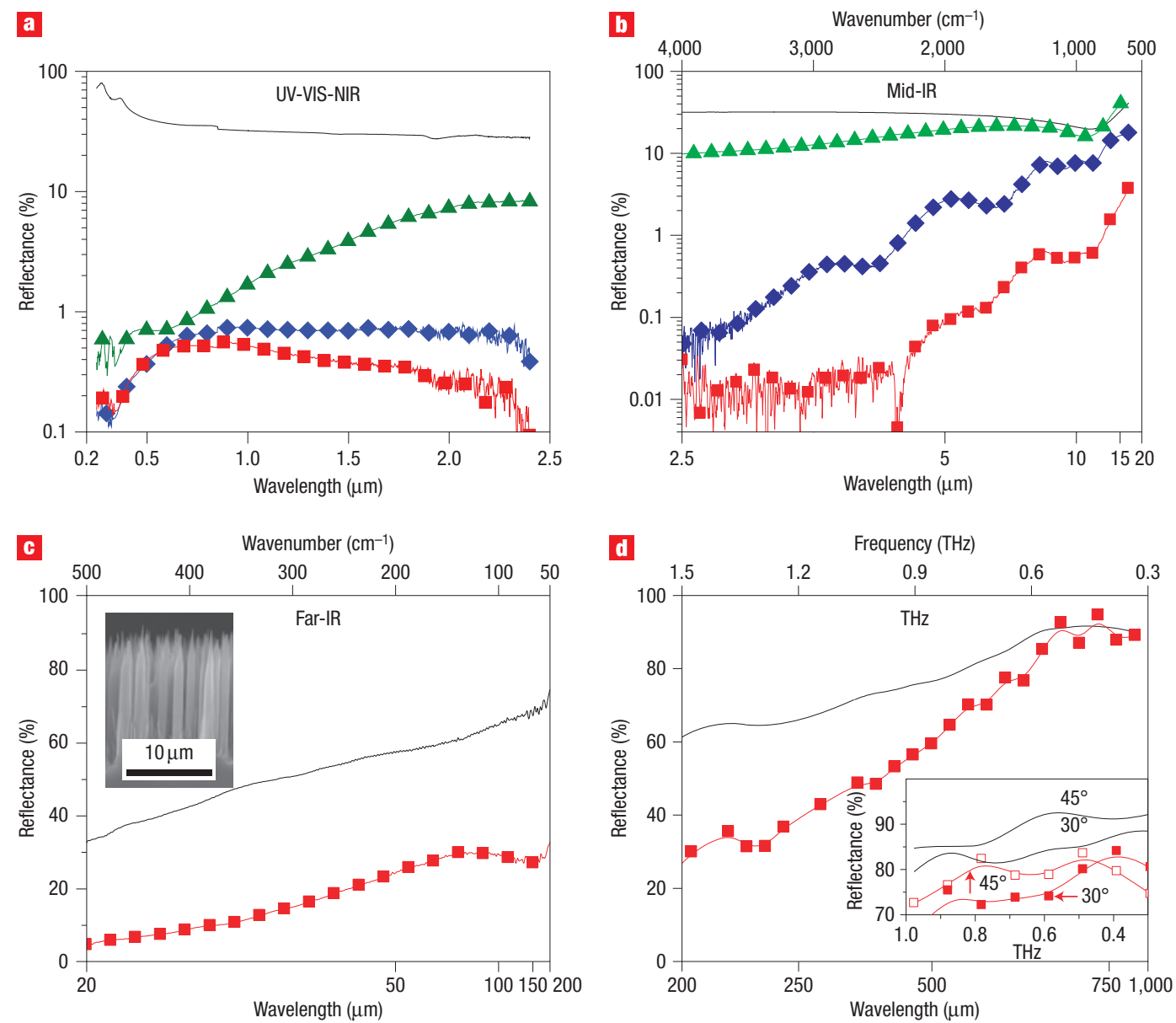

Figure 2 Broadband anti-reflection properties of silicon nanotips (SiNTs). a, Comparison of the hemispherical reflectance (using IS) as a function of wavelength for a planar Si wafer (solid line, black) and SiNTs (symbols) for $L=1.6 \mu \mathrm{m}$ (green), $5.5 \mu \mathrm{m}$ (blue) and $16 \mu \mathrm{m}$ (red) at UV, VIS and NIR wavelengths. $\mathbf{b}$, Comparison of the specular reflectance (without IS) as a function of wavelength in the mid-infrared region for an AOl of $30^{\circ}$. $\mathbf{c}, \mathbf{d}$, Comparison of specular reflectance as a function of wavelength for a planar silicon wafer (solid line, black) and SiNTs with $L=16 \mu \mathrm{m}$ (red) in the far-infrared (c) and terahertz (d) regions for an A0I of $30^{\circ}$. Inset in c shows the cross-sectional SEM image of the $L=16 \mu \mathrm{m}$ SiNTs. Inset in $\mathbf{d}$ compares the reflectance in planar silicon (solid line, black) and SiNTs (symbols, red) with unpolarized light and an $\mathrm{AOI}$ of $30^{\circ}$ (filled squares) and $45^{\circ}$ (open squares). The solid red lines in the inset of $\mathbf{d}$ are guides to the eye. 

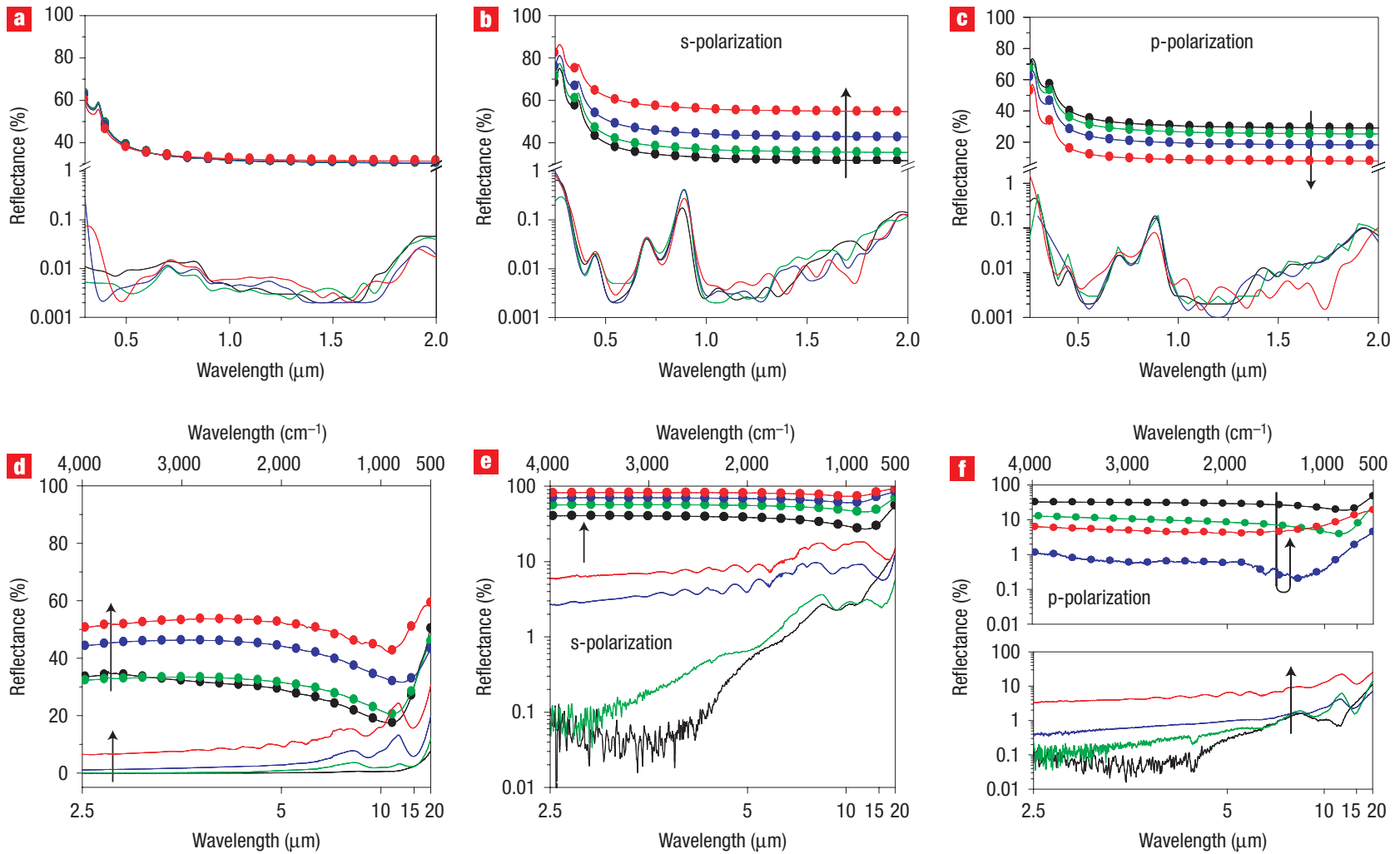

Figure 3 Angle of incidence and polarization-dependent anti-reflection properties of silicon nanotips (SiNTs). a-c, Comparison of the specular reflectance from planar Si wafer (symbols) and SiNTs (solid lines), with $L=16 \mu \mathrm{m}$, as a function of wavelength for unpolarized (a), s-polarized (b) and p-polarized (c) light at wavelengths below $2 \mu \mathrm{m}$ for four different values of AOl: $5^{\circ}$ (black), $30^{\circ}$ (green), $45^{\circ}$ (blue) and $60^{\circ}$ (red). Note that the $y$-axis is linear for planar Si wafer data and logarithmic for the SiNT data. $\mathbf{d}-\mathbf{f}$, Comparison of the specular reflectance from a planar Si wafer (solid lines) and SiNTs (symbols), with $L=16 \mu \mathrm{m}$, as a function of wavelength for unpolarized (d), s-polarized (e) and p-polarized (f) light at wavelengths between 2.5 and $20 \mu \mathrm{m}$ for four different values of A0l: $30^{\circ}$ (black), $60^{\circ}$ (green), $75^{\circ}$ (blue) and $80^{\circ}$ (red). Note that the $y$-axis for each panel is different, and that the planar Si and SiNT data in $\mathrm{f}$ are drawn in separate graphs for clarity. The direction of the arrows indicates the variation in reflectance as the angles of incidence were increased.

The anti-reflection performance of silicon in the long wavelength range is relatively poor. In this context, SiNTs demonstrate an improved anti-reflection property as a function of wavelength in the region $\sim 0.3-1,000 \mu \mathrm{m}$ (Fig. 2). Hemispherical reflectances (diffuse + specular) of SiNTs using the integrating sphere (IS) are shown in Fig. 2a. The results show that long $(>5 \mu \mathrm{m})$ SiNTs have an average reflectance of $<1 \%$ over $0.5-2.5 \mu \mathrm{m}$ range. However, more importantly, we demonstrate an average hemispherical reflectance of $0.2 \%$ in the $0.25-0.4 \mu \mathrm{m}$ range, which is the least reported to date, to the best of our knowledge. The hemispherical reflectance of SiNTs (length $L$ of $\sim 1.6 \mu \mathrm{m}$ ) will increase slightly beyond $1,100 \mathrm{~nm}$ because of the decrease in the absorbed component of the light below the indirect bandgap of Si $(1,100 \mathrm{~nm})^{21}$. The best specular reflectance values (without IS) for SiNTs are below 2 and 30\% for the mid-IR and far-IR ranges, respectively (Fig. 2b,c). A comparison of the specular and hemispherical reflectance in the UV-VIS-NIR region (see Supplementary Information, Fig. S1) has been made to test the effect of the random nature of these SiNTs. Figure $2 \mathrm{~d}$ demonstrates the results of the anti-reflection properties of SiNTs when put through the ultimate test in the terahertz region $(\mathrm{THz}$, $200-1,000 \mu \mathrm{m})$ in the s-polarized mode with $\mathrm{AOI}=30^{\circ}$. The reflectance in the $16-\mu \mathrm{m}$-long SiNTs is less than that of planar silicon by at least $10 \%$ in the $0.7-1.5 \mathrm{THz}$ regime, but the reflectance values in the two converged near $0.3 \mathrm{THz}(1,000 \mu \mathrm{m})$ mark. The inset in Fig. 2d confirms a suppression of reflectance for unpolarized light in the $\mathrm{THz}$ regime in the SiNTs compared to planar silicon under $30^{\circ}$ and $45^{\circ}$ AOI (see also Supplementary Information, Fig. S2, for the data in the $\mathrm{THz}$ regime using polarized light). Although most of the lithographically prepared ordered sub-wavelength structures fail in this regime, the aperiodic SiNT structure clearly shows its unusual capacity in subduing the reflectance over the extended wavelength regime of $0.3-1,000 \mu \mathrm{m}$ in the unpolarized mode. A better anti-reflection performance may be possible in this $\mathrm{THz}$ range with further optimization of the nanotip length as described later.

In order to perform as a good anti-reflector, the structure must show low reflectance over a wide range of AOI values for both forms of light polarization, s and p (ref. 30). This is shown in Fig. 3 for 16- $\mu \mathrm{m}$-long SiNTs. First, the reflectance from planar silicon and SiNTs surfaces were compared using unpolarized light at wavelength ranges $0.3-2.2 \mu \mathrm{m}$ and $2.5-20 \mu \mathrm{m}$, respectively. Generally, for the polished Si substrate the specular reflectance varies strongly with AOI for both s- and p-polarized light (Fig. 3b,c). In contrast, SiNTs are not highly sensitive to polarization; the specular reflectance of the 16- $\mu \mathrm{m}$-long SiNTs remains below $1 \%$ at $\mathrm{AOI}$ values between $5^{\circ}$ and $60^{\circ}$ for both $\mathrm{s}$ - and p-polarized light (Fig. 3b,c) in the UV-VIS-NIR region. In the mid-IR range the reflectance is below $20 \%$ for the same sample with s- and p-polarized light within the AOI range of 

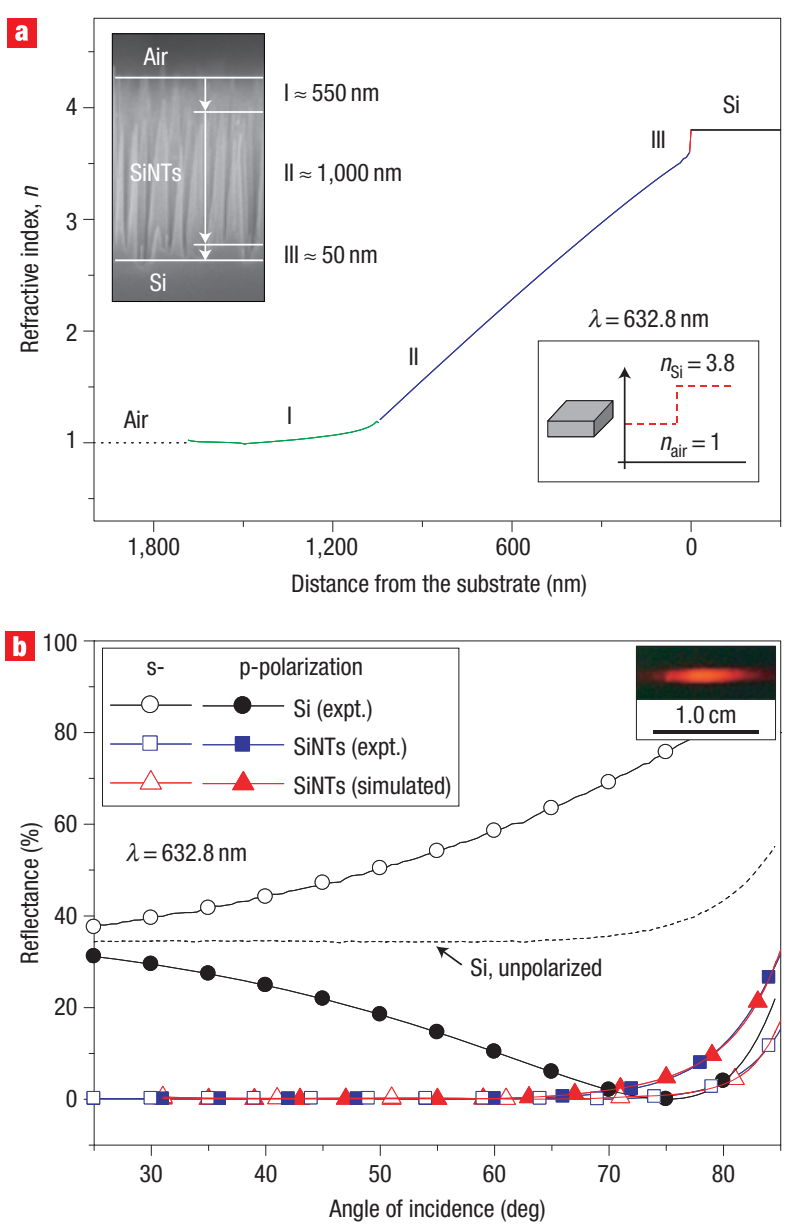

Figure 4 Gradient refractive-index profile simulation of the surface of the SiNTs. a, Refractive-index profile of the SiNT surface. Three distinct regions (described in the text) were observed: region I with refractive index close to 1.0, region II with a graded index, and region III with refractive index close to 3.8. The top left inset shows a cross-sectional SEM image of the SiNTs showing the three distinct regions. The bottom right inset shows the refractive-index profile of the polished Si wafer, for reference. $\mathbf{b}$, The reflectance of the Si wafer and the SiNTs $(L=1.6 \mu \mathrm{m})$ as a function of angle of incidence using s- and p-polarized light of $632.8 \mathrm{~nm}$ wavelength; the line joining the data points as well as the symbols (red) represent the simulated (calculated) reflectance data for SiNTs $(L=1.6 \mu \mathrm{m})$ using the profile in $\mathbf{a}$. The top right inset in $\mathbf{b}$ shows the optical image of the laser spot at an $\mathrm{AOI}$ of $85^{\circ}$. The measured reflectance data for planar Si with unpolarized light are also shown.

$30-80^{\circ}$ (Fig. 3e,f). For the sake of comparison, the reflectance versus wavelength data using polarized light for three different lengths of nanotip samples have been provided at AOI values of $30^{\circ}$ and $60^{\circ}$ (see Supplementary Information, Fig. S3). Barring monochromatic measurements, most sub-wavelength or quarterwavelength structures, independent of the material used, show a suppression of reflectance below an $\mathrm{AOI}$ of $60^{\circ}$ (in an extended VIS to IR region) $)^{16,21,23,31-34}$, whereas in this case the suppression could easily be observed up to $70^{\circ}$. The reflectance from the aperiodic SiNTs demonstrates a minimum sensitivity to the angle of incidence compared with periodic multilayer coatings and subwavelength structures, as well as non-periodic porous materials.

In order to interpret the wide angle and polarizationindependent anti-reflection properties, the gradient index of refraction was applied to the SiNTs and the refractive-index profile determined by theoretical analyses ${ }^{35}$ and simulated fitting (WVASE, see Methods and Supplementary Information, Fig. S4). The best refractive-index profile to simulate the measured data was only generated (Fig. 4a) for a SiNT array of length $1.6 \mu \mathrm{m}$, by assuming three layers of $\mathrm{Si}$, instead of one, having graded refractive index. Three distinct regions appear in the refractiveindex profile to fit the reflectance spectra as shown in Fig. 4b. Region I is near the apex of the SiNTs, and serves as the airSiNT interface and covers about $550 \mathrm{~nm}$ from the top of the SiNTs. Region II has a depth of $\sim 1,000 \mathrm{~nm}$ from the bottom of Region I and constitutes the bulk of the SiNTs. Region III extends about $50 \mathrm{~nm}$ from the base of the SiNTs and serves as the SiNTs-Si interface. Regions I and III, having values of refractive index close to that of air (1.0) and Si (3.8 at $632.8 \mathrm{~nm})$, respectively, behave as the two limits of the refractive-index profile of the SiNTs. Region II has a refractive index graded with the volume fraction of Si as one goes deeper into the SiNTs.

In contrast with the gradient refractive index obtained in multilayered coatings or multi-material structures ${ }^{17,34}$, the SiNTs result in a smooth profile with only a single element, with silicon having the optimum structure. Defining volume fraction as the ratio of $\mathrm{Si}$ to the total volume, we estimate that $\mathrm{Si}$ makes up $<0.5$ of the volume of the whole SiNTs array having different nanotip densities. Using the Bruggeman effective medium approximation $^{36}$, a reasonable effective refractive index of the SiNTs was found to be between 1.6 and 1.8. The improved flatness of the refractive index profile near the air-silicon interface $^{35}$ is key to anti-reflection performance. Longer apexes (Region I) of the SiNTs have been shown to reduce reflection both as a function of the AOI and wavelength (see Supplementary Information, Fig. S5). We believe these nanotip structures are not fully optimized. Taking our cue from grating structures with groove depths exceeding $30 \mu \mathrm{m}$, conventionally used in the THz regime $e^{37,38}$, we believe that longer nanotips would perform better in the $\mathrm{THz}$ regime as they would have a finer refractive-index gradient at the air-solid interface. However, we did not observe any loss of anti-reflection performance at short wavelengths due to increasing lengths of the SiNTs (Fig. 2a; see also Supplementary Information, Fig. S5). In conclusion, the excellent anti-reflection properties observed in the SiNTs are close to near-ideal anti-reflection coatings ${ }^{15,35}$.

\section{METHODS}

\section{FABRICATION OF SILICON NANOTIP ARRAYS}

Uniform arrays of aligned SiNTs were formed on $\mathrm{n}^{+}$-type $(\rho=0.001-0.005$

$\Omega$-cm) single-crystalline silicon (100) wafers by a high-density electron cyclotron resonance (ECR) plasma etching using reactive gases comprising silane $\left(\mathrm{SiH}_{4}\right)$, methane $\left(\mathrm{CH}_{4}\right)$, hydrogen $\left(\mathrm{H}_{2}\right)$ and argon (Ar). A detailed description of SiNT formation using the self-masked dry etching technique can be found elsewhere ${ }^{27-29}$.

\section{OPTICAL MEASUREMENTS}

Optical reflectance measurements on SiNTs were carried out using five optical measurement systems. A JASCO-V570 (UV-VIS-NIR) spectrophotometer attached with an integrating sphere was used for the reflectance/absorption measurements in the UV-VIS-NIR range. The specular reflectance and polarization-dependent reflectance as a function of wavelength $(250-2,000 \mathrm{~nm})$ and various angles of incidence $\left(5-60^{\circ}\right)$ were measured with a JASCO ARN-475 spectral measurement accessory and ARG- 476 polarizer. However, care was taken to ensure that the sample size $\left(3 \times 3 \mathrm{~cm}^{2}\right)$ far exceeded the elliptical spot size (Fig. 4b, inset) at large AOI values.

A stabilized helium-neon laser $(632.8 \mathrm{~nm})$ with frequency stability of $\pm 5 \mathrm{MHz}$ was used to measure the reflectance-incident angle spectrum $\left(25-85^{\circ}\right)$. The angular resolution of the instrument was $0.01^{\circ}$.

A Thermo Nicolet 6700-Fourier transform infrared (FTIR) spectroscope was used for the mid-IR region $\left(4,000-500 \mathrm{~cm}^{-1}\right)$ (Fig. 2b,c), which had a resolution 
of $4 \mathrm{~cm}^{-1}$ and uses a deuterated tryglycine sulphate (DTGS) detector. The system had a Spectra-Tech Model 500 Variable Angle Specular Reflectance accessory $\left(20-85^{\circ}\right)$ and infrared polarizer. A Bruker IFS $66 \mathrm{v} / \mathrm{s}$ FTIR spectroscope was used for the far-IR $\left(500-50 \mathrm{~cm}^{-1}\right)$ spectral region, with an FIR deuterated tryglycine sulphate/polyethylene (DTGS/PE) detector and Hg light source.

The measurement in the $\mathrm{THz}$ regime used a reflective-type $\mathrm{THz}$-time domain spectroscopy (THz-TDS) system with a THz incident angle of $30^{\circ}$. We used 1-mm-thick ZnTe as an emitter with $100 \mathrm{~mW}$ Ti:sapphire laser excitation. The detector was a dipole-type photoconductive antenna fabricated on a low-temperature GaAs substrate with $5-\mu \mathrm{m}$ gap in the centre and $30-\mu \mathrm{m}$ dipole length, which was gated by a $30 \mathrm{~mW}$ gating laser.

\section{OPTICAL MODELLING AND SIMULATION}

Assuming a single graded layer between air and substrate, three types of gradientindex profile were constructed using linear, exponential and exponential sine variations ${ }^{35}$ using an optical software (WVASE 32, J.A. Woolam; see Supplementary Information, Fig. S4a,c,e). The same software was used to simulate the reflectance data (see Supplementary Information, Fig. S4b,d,f). However, the best fit to the measured reflectance data was obtained with a profile constructed assuming three graded layers using this software.

Received 31 August 2007; accepted 2 November 2007;

published 2 December 2007.

References

1. Lee, L. P. \& Szema, R. Inspirations from biological optics for advanced photonic systems. Science 310 $1148-1150$ (2005).

2. Vukusic, P. \& Sambles, J. R. Photonic structures in biology. Nature 424, 852-855 (2003).

3. Parker, A. R. \& Townley, H. E. Biomimetics of photonic nanostructures. Nature Nanotech. 2 347-353 (2007).

4. Potyrailo, R. A. et al. Morpho butterfly wing scales demonstrate highly selective vapour response. Nature Photon. 1, 123-128 (2007).

5. Neinhuis, C. \& Barthlott, W. Characterisation and distribution of water-repellent, self-cleaning plant surfaces. Ann. Bot. 79, 667-677 (1997).

6. Ghiradella, H. et al. Ultraviolet reflection of a male butterfly: Interference color caused by thin-layer elaboration of wing scales. Science 178, 1214-1217 (1972).

7. Aizenberg, J. et al. Calcitic microlenses as part of the photoreceptor system in brittlestars. Nature 412 819-822 (2001).

8. Bernhard, C. G. Structural and functional adaptation in a visual system. Endeavour 26, 79-84 (1967).

9. Srinivasarao, M. Nano-optics in the biological world: beetles, butterflies, birds, and moths. Chem Rev. 99, 1935-1961 (1999).

10. Feng, L. et al. Super-hydrophobic surfaces: From natural to artificial. Adv. Mater. 14, 1857-1860 (2002).

11. Gu, Z. Z. et al. Structural color and the lotus effect. Angew. Chem. Int. Edn 42, 894-897 (2003).

12. Groning, P. Nanotechnology: An approach to mimic natural architectures and concepts. Adv. Eng. Mater. 7, 279-291 (2005).

13. Clapham, P. B. \& Hutley, M. C. Reduction of lens reflection by moth eye principle. Nature $\mathbf{2 4 4}$, 281-282 (1973).

14. Wilson, S. J. \& Hutley, M. C. The optical-properties of moth eye antireflection surfaces. Optica Act 29, $993-1009$ (1982).

15. Southwell, W. H. Pyramid-array surface-relief structures producing antireflection index matching on optical-surfaces. J. Opt. Soc. Am. A 8, 549-553 (1991).
16. Grann, E. B. Moharam, M. G. \& Pommet, D. A. Optimal-design for antireflective tapered twodimensional subwavelength grating structures. J. Opt. Soc. Am. A 12, 333-339 (1995).

17. Xi, J. Q. et al. Optical thin-film materials with low refractive index for broadband elimination of Fresnel reflection. Nature Photon. 1, 176-179 (2007).

18. Lee, C. et al. A novel silicon nanotips antireflection surface for the micro sun sensor. Nano Lett. 5 2438-2442 (2005)

19. Chattopadhyay, S., Chen, L. C. \& Chen, K. H. Nanotips: Growth, model, and applications. Crit. Rev. Solid State Mater. Sci. 31, 15-53 (2006).

20. Striemer, C. C. \& Fauchet, P. M. Dynamic etching of silicon for broadband antireflection applications. Appl. Phys. Lett. 81, 2980-2982 (2002).

21. Kanamori, Y., Sasaki, M. \& Hane, K. Broadband antireflection gratings fabricated upon silicon substrates. Opt. Lett. 24, 1422-1424 (1999).

22. Kanamori, Y. et al. $100 \mathrm{~nm}$ period silicon antireflection structures fabricated using a porous alumina membrane mask. Appl. Phys. Lett. 78, 142-143 (2001).

23. Yu, Z. et al. Fabrication of large area subwavelength antireflection structures on Si using trilayer resist nanoimprint lithography and liftoff. J. Vac. Sci. Technol. B 21, 2874-2877 (2003).

24. Zaidi, S. H., Ruby, D. S. \& Gee, J. M. Characterisation of random reactive ion etched-textured silicon solar cells. IEEE Trans. Electron Devices 48, 1200-1206 (2001).

25. Kanamori, Y., Ishimori, M. \& Hane, K. High efficient light-emitting diodes with antireflection subwavelength gratings. IEEE Photon. Technol. Lett. 14, 1064-1066 (2002).

26. Glaser, T. et al. High temperature resistant antireflective moth-eye structures for infrared radiation sensors. Microsyst. Technol. 11, 86-90 (2005).

27. Hsu, C. H. et al. Generally applicable self-masked dry etching technique for nanotip array fabrication. Nano Lett. 4, 471-475 (2004).

28. Chen, K. H. et al. Method of forming a nanotip array in a substrate by forming masks on portions of the substrate and etching the unmasked portions. US patent 6,960,528 B2 (2005).

29. Hsu, C. H. et al. Morphology control of silicon nanotips fabricated by electron cyclotron resonance plasma etching. J. Vac. Sci. Technol. B 24, 308-311 (2006).

30. Macleod, H. A. in Thin Film Optical Filters 3rd edn (Taylor \& Francis, London, 2001).

31. Minot. M. J. The angular reflectance of single-layer gradient refractive-index films. J. Opt. Soc. Am. 67, 1046-1050 (1977).

32. Dobrowolski, J. A. \& Piotrowski, S. H. C. Refractive index as a variable in the numerical design of optical thin film systems. Appl. Opt. 21, 1502-1511 (1982).

33. Lalanne, P. \& Morris, G. M. Antireflection behavior of silicon subwavelength periodic structures for visible light. Nanotechnology 8, 53-56 (1997)

34. Dobrowolski, J. A. et al. Toward perfect antireflection coatings. 3. Experimental results obtained with the use of Reststrahlen materials. Appl. Opt. 45, 1555-1562 (2006).

35. Poitras, D. et al. Toward perfect antireflection coatings. 2. Theory. Appl. Opt 43, 1286- 1295 (2004).

36. Tompkins, H. G. in A User's Guide to Ellipsometry (Academic Press, New York, 1999).

37. Gatesman, A. J. et al. An anti-reflection coating for silicon optics at terahertz frequencies. IEEE Microw. Guid. Wave Lett. 10, 264-266 (2000).

38. Brückner, C. et al. Broadband antireflective surface-relief structure for $\mathrm{THz}$ optics. Opt. Express 15, 779-789 (2007).

\section{Acknowledgements}

We acknowledge financial support from the Ministry of Education under the ATU plan and the Nationa Science Council in Taiwan, the Air Force Office of Scientific Research, the Asian Office of Aerospace Research and Development and the US Army Research Office in the Far East.

Correspondence and requests for materials should be addressed to S.C. and L.C.C.

Supplementary information accompanies this paper on www.nature.com/naturenanotechnology.

\section{Author contributions}

Y.F.H. conceived and performed the UV-VIS, IR and angle-dependent experiments; H.C.L. and C.H.H. synthesized the SiNTs; Y.H.C. and C.S.L. performed the far IR measurements; T.A.L. Y.K.H. and C.L.P. performed the THz measurements; Y.J.J and C.Y.P. carried out the theoretical analyses; Y.F.H., S.C. K.H.C. and L.C.C. discussed and interpreted the result; and S.C., K.H.C. and L.C.C. co-wrote the paper.

Reprints and permission information is available online at http://npg.nature.com/reprintsandpermissions/ 